University of Nebraska - Lincoln

DigitalCommons@University of Nebraska - Lincoln

\title{
Surface complexation modeling for predicting solid phase arsenic concentrations in the sediments of the Mississippi River Valley alluvial aquifer, Arkansas, USA
}

\author{
Md. Salah U. Sharif \\ University of Arkansas, sharif.msalah@gmail.com \\ Ralph K. Davis \\ University of Arkansas \\ Kenneth F. Steele \\ University of Arkansas \\ Burmshik Kim \\ University of Arkansas \\ Phillip D. Hays \\ University of Arkansas \\ See next page for additional authors \\ Follow this and additional works at: https://digitalcommons.unl.edu/usgsstaffpub \\ Part of the Earth Sciences Commons
}

Sharif, Md. Salah U.; Davis, Ralph K.; Steele, Kenneth F.; Kim, Burmshik; Hays, Phillip D.; Kresse, Tim M.; and Fazio, John A., "Surface complexation modeling for predicting solid phase arsenic concentrations in the sediments of the Mississippi River Valley alluvial aquifer, Arkansas, USA" (2011). USGS Staff -Published Research. 518.

https://digitalcommons.unl.edu/usgsstaffpub/518

This Article is brought to you for free and open access by the US Geological Survey at DigitalCommons@University of Nebraska - Lincoln. It has been accepted for inclusion in USGS Staff -- Published Research by an authorized administrator of DigitalCommons@University of Nebraska - Lincoln. 


\section{Authors}

Md. Salah U. Sharif, Ralph K. Davis, Kenneth F. Steele, Burmshik Kim, Phillip D. Hays, Tim M. Kresse, and John A. Fazio 


\title{
Surface complexation modeling for predicting solid phase arsenic concentrations in the sediments of the Mississippi River Valley alluvial aquifer, Arkansas, USA
}

\author{
Tim M. Kresse ${ }^{\mathrm{b}}$, John A. Fazio ${ }^{\mathrm{c}}$ \\ a Environmental Dynamics Program, Department of Geosciences, University of Arkansas, Fayetteville, AR 72701, USA \\ ${ }^{\mathrm{b}}$ US Geological Survey, Water Science Center, 401 Hardin Rd., Little Rock, AR 72211, USA

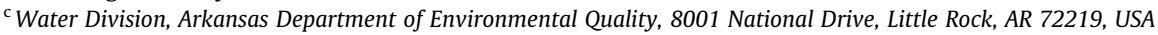

Md. Salah U. Sharif ${ }^{\text {a,* }}$, Ralph K. Davis ${ }^{a}$, Kenneth F. Steele ${ }^{a}$, Burmshik Kim ${ }^{\text {a }}$, Phillip D. Hays ${ }^{\text {a }}$,

\section{A R T I C L E I N F O}

Article history:

Available online 6 January 2011

\begin{abstract}
A B S T R A C T
The potential health impact of As in drinking water supply systems in the Mississippi River Valley alluvial aquifer in the state of Arkansas, USA is significant. In this context it is important to understand the occurrence, distribution and mobilization of As in the Mississippi River Valley alluvial aquifer. Application of surface complexation models (SCMs) to predict the sorption behavior of As and hydrous Fe oxides (HFO) in the laboratory has increased in the last decade. However, the application of SCMs to predict the sorption of As in natural sediments has not often been reported, and such applications are greatly constrained by the lack of site-specific model parameters. Attempts have been made to use SCMs considering a component additivity (CA) approach which accounts for relative abundances of pure phases in natural sediments, followed by the addition of SCM parameters individually for each phase. Although few reliable and internally consistent sorption databases related to HFO exist, the use of SCMs using laboratory-derived sorption databases to predict the mobility of As in natural sediments has increased. This study is an attempt to evaluate the ability of the SCMs using the geochemical code PHREEQC to predict solid phase As in the sediments of the Mississippi River Valley alluvial aquifer in Arkansas. The SCM option of the double-layer model (DLM) was simulated using ferrihydrite and goethite as sorbents quantified from chemical extractions, calculated surface-site densities, published surface properties, and published laboratory-derived sorption constants for the sorbents. The model results are satisfactory for shallow wells ( $10.6 \mathrm{~m}$ below ground surface), where the redox condition is relatively oxic or mildly suboxic. However, for the deep alluvial aquifer (21-36.6 m below ground surface) where the redox condition is suboxic to anoxic, the model results are unsatisfactory.
\end{abstract}

(c) 2011 Elsevier Ltd. All rights reserved.

\section{Introduction}

The movement and transport of As in groundwater can be significantly slowed or enhanced by chemical or biogeochemical reactions occurring at mineral-water interfaces. In general, physico-chemical processes such as precipitation and dissolution, surface complexation, and ion exchange determine the interaction of As with solid sorbent surfaces (Figueira da Silva, 2005). The most common and dominant As attenuation reaction is surface complexation with hydrous metal oxides (Sracek et al., 2004; Langmuir, 1997; Korte and Fernando, 1991; Shevenell et al., 1999). In theory, surface complexation models (SCMs) are based on fundamental chemical and physical principles that are controlled by measurable parameters such as specific surface area, surface-site density, elec-

* Corresponding author. Present address: Ecometrix Incorporated, 6800 Campobello Road, Mississauga, Ontario, Canada L5N 2L8. Tel.: +1 647346 3695; fax: +1 9057942338 .

E-mail address: sharif.msalah@gmail.com (Md.S.U. Sharif). trical and chemical potentials of the surfaces, and intrinsic surface constants associated with reactive surface sites and adsorbate ions, etc. (Figueira da Silva, 2005; Zachara and Westall, 1999; Miller, 2000; Dzombak and Morel, 1990; Davis et al., 2004). Fundamental sorption reactions that take place at metal oxide surfaces are fully describable using surface complexation theory (Dzombak and Morel, 1990; Hemond, 1995; Goldberg, 1998; Koretsky, 2000; Sposito, 1989).

Scientific literature documents considerable experimental data for As and other trace elements in systems with one mineral phase, and SCMs have been applied to accurately describe these data (Dzombak and Morel, 1990; Goldberg, 1998; Swedlund and Webster, 1999; Dixit and Hering, 2003, 2006; Ali and Dzombak, 1996; Davis et al., 1978; Westall and Hohl, 1980; Belzile and Tessier, 1990; Manning and Goldberg, 1996, 1997; Sadiq, 1997). The application of SCMs to natural sediments of varying mixtures of minerals is difficult because of the presence of secondary minerals and organic coatings (Davis et al., 2004; Coston et al., 1995; Padmanabhan and Mermut, 1996; Penn et al., 2001). A component 
additivity (CA) approach is commonly used for SCMs, where it is assumed that a mineral assemblage is composed of a mixture of one or more pure phases, whose surface chemical reactions are known from independent experiments of each phase (Davis et al., 1998, 2004; Cowan et al., 1992; Turner et al., 1996; Arnold et al., 2001; Prikryl et al., 2001). The next step is to measure the relative amounts of surface areas of each mineral present in the soils or sediments. Then, adsorption of the mixture of mineral phases can be predicted by an equilibrium calculation, without any fitting of experimental data for the mixture (Honeyman, 1996). In the CA approach, it is commonly assumed that one mineral phase dominates adsorption (Davis et al., 1998; Schmeide et al., 2000; Barnett et al., 2002; Waite et al., 2000), which facilitates a straightforward equilibrium calculation if the exposed surface area and surface-site density of that mineral phase in the soil or sediment can be quantified. SCMs typically use a combination of minerals with varying surface properties in order to describe the formation of surface complexes of elements present in groundwater on different mineral surfaces.

Successful SCM simulation depends on appropriate selection of internally consistent intrinsic surface constants, accurate chemical analysis of groundwater, and proper quantification of sorbents and their surface properties in each sediment sample. Although many published sorption databases related to As and other species on different sorbent surfaces exist (Dixit and Hering, 2003, 2006; Manning and Goldberg, 1996; Sigg, 1979; Appelo et al., 2002; Van Geen et al., 1994), few reliable and internally consistent sorption databases are available to quantify all of the surface species of interest and relevant parameters of the models (Dzombak and Morel, 1990; Sverjensky and Sahai, 1996; Sahai and Sverjensky, 1997a,b). This situation has changed somewhat in recent years with the increased availability of sorption data for surface reactions related to As and other elements on different metal oxide surfaces. However, little information has been reported on the predictability and applicability of SCMs to complex natural sediments (Miller, 2000, 2001; Davis et al., 1998, 2004; Appelo et al., 2002; US Geological Survey, 1994; Wang et al., 1997; Turner and Pabalan, 1999; Tessier et al., 1996; Fukushi and Sato, 2005). A review of the literature indicates that the use of SCMs to predict the environmental mobility of As or other trace metals in groundwater has been limited (Dzombak and Morel, 1990; Tessier et al., 1996; Miller, 2001; Smith and Jenne, 1991). Geochemist's Workbench (Bethke, 2006), PHREEQC (Parkhurst and Appelo, 1999), and MINTEQA2 (Allison et al., 1991) are the most versatile of the presently available geochemical codes that use SCMs for incorporating multiple levels of uncertainty.

In general, impacts from As to municipal-supply water systems in Arkansas are low as most of the municipal-supply water systems abstract water from deeper Tertiary aquifers where As is usually $<0.5 \mu \mathrm{g} / \mathrm{L}$. It has been reported that irrigation water wells completed in the shallow (25-30 m below ground surface) Mississippi River Valley alluvial aquifer in southeastern Arkansas has As concentrations $(<0.5-77 \mu \mathrm{g} / \mathrm{L})$ exceeding the US EPA standard of $10 \mu \mathrm{g} / \mathrm{L}$ (Kresse and Fazio, 2002, 2003; Sharif, 2007; Sharif et al., 2008a,b). Approximately 200 public water supply wells draw water from the shallow Mississippi River Valley alluvial aquifer serving about 450,000 people. So, the potential health impact of As to drinking water supply systems in Arkansas is still significant. Hydrogeochemical data and the redox environment in the Mississippi River Valley alluvial aquifer suggest reductive dissolution of HFO as the dominant As release mechanism (Kresse and Fazio, 2002, 2003; Sharif, 2007; Sharif et al., 2008a,b). Gypsum solubility and simultaneous $\mathrm{SO}_{4}^{2-}$ reduction with co-precipitation of As and sulfide is an important limiting process controlling the concentration of dissolved As in groundwater. Spatial and temporal variability of As in groundwater is controlled by spatial distribution and the redox state of individual redox zones in the aquifer, which is controlled by recharge potential, permeability of the surface aquitard, intensity of irrigation, and local flow dynamics in the aquifer. The redox state is the primary control on the rate of HFO reduction and the amount of As in groundwater (Sharif et al., 2008a,b).

This paper is part of a comprehensive research program with a primary goal of understanding the distribution and mobilization of As in groundwater in the Mississippi River Valley alluvial aquifer (Bayou Bartholomew watershed) of southeastern Arkansas. The purpose of this paper is to evaluate the ability of the SCMs based on a CA approach using DLM in the geochemical code PHREEQC to predict solid phase As occurrence in the sediments collected from six boreholes drilled into the Mississippi River Valley alluvial aquifer. The DLM was used with a number of adjustable or measured parameters, including the stability or equilibrium constants, total site concentration of reactive surface hydroxyl groups, total specific surface area, total solid concentration, and water-mineral ratio.

\section{Study area}

The study area, approximately $225 \mathrm{~km}^{2}$, is located in the southern part of Jefferson County, Arkansas (Fig. 1). It is bounded by the Arkansas River to the NE and Bayou Bartholomew to the SW. The area comprises the northeastern part of the Bayou Bartholomew watershed and is entirely covered by Holocene alluvial deposits of the Mississippi and Arkansas rivers. The Holocene alluvial deposits are represented by a downward-coarsening sequence from clays, silts and fine sands at the surface (herein referred to as the surface aquitard), to coarse sands and gravels at the base. Pleistocene alluvial deposits of the Mississippi and Arkansas rivers form terraces with minor exposures of Tertiary-age strata along topographically high areas, and are found beyond the western part of the study area (Kresse and Fazio, 2002). The thickness of the surface aquitard varies from $<6$ to $12 \mathrm{~m}$. The thickness of the alluvial aquifer ranges from 18 to $43 \mathrm{~m}$ (Kleiss et al., 2000). An assemblage of channel fill, point bar, and back-swamp deposits associated with present and former channels of the Mississippi and Arkansas rivers produced heterogeneity in lithology and resulted in large spatial and vertical variations in the hydraulic properties within the aquifer system (Joseph, 1999). The regional direction of groundwater flow is generally to the south and east except where affected by intense groundwater withdrawals (Schrader, 2001). Row-crop agriculture represents the major land use in the floodplain, whereas silviculture dominates the land use in the terrace portion of the watershed. Eastern Arkansas receives an annual precipitation of 1.2-1.4 m (Freiwald, 1985).

\section{Materials and methods}

Three pairs of nested monitoring wells (10.6 and $36.6 \mathrm{~m}$ deep) were drilled, installed and developed at three contrasting sites selected as a high As ( $>50 \mu \mathrm{g} / \mathrm{L}$ ) area in the NW (DRL1), a medium As $(10-50 \mu \mathrm{g} / \mathrm{L})$ area in the south (DRL2), and a low As $(<10 \mu \mathrm{g} / \mathrm{L})$ area in the NE (DRL6). The capital letters " $D$ " and "S" are used after the site designation letters (DRL1, DRL2 or DRL6) to describe deep and shallow monitoring wells, respectively.

The materials and methods consist of collection and preservation of soil cuttings (Sharif et al., 2008a,b), preparation of sediment samples for a modified 5-step sequential extraction procedure (Tessier et al., 1979; Chao and Zhou, 1983), X-ray diffraction (XRD) and scanning electron microscopy (SEM) analysis, groundwater sampling, field monitoring and laboratory analyses. The following section is excerpted from previous papers (Sharif, 2007; Sharif et al., 2008a,b). 


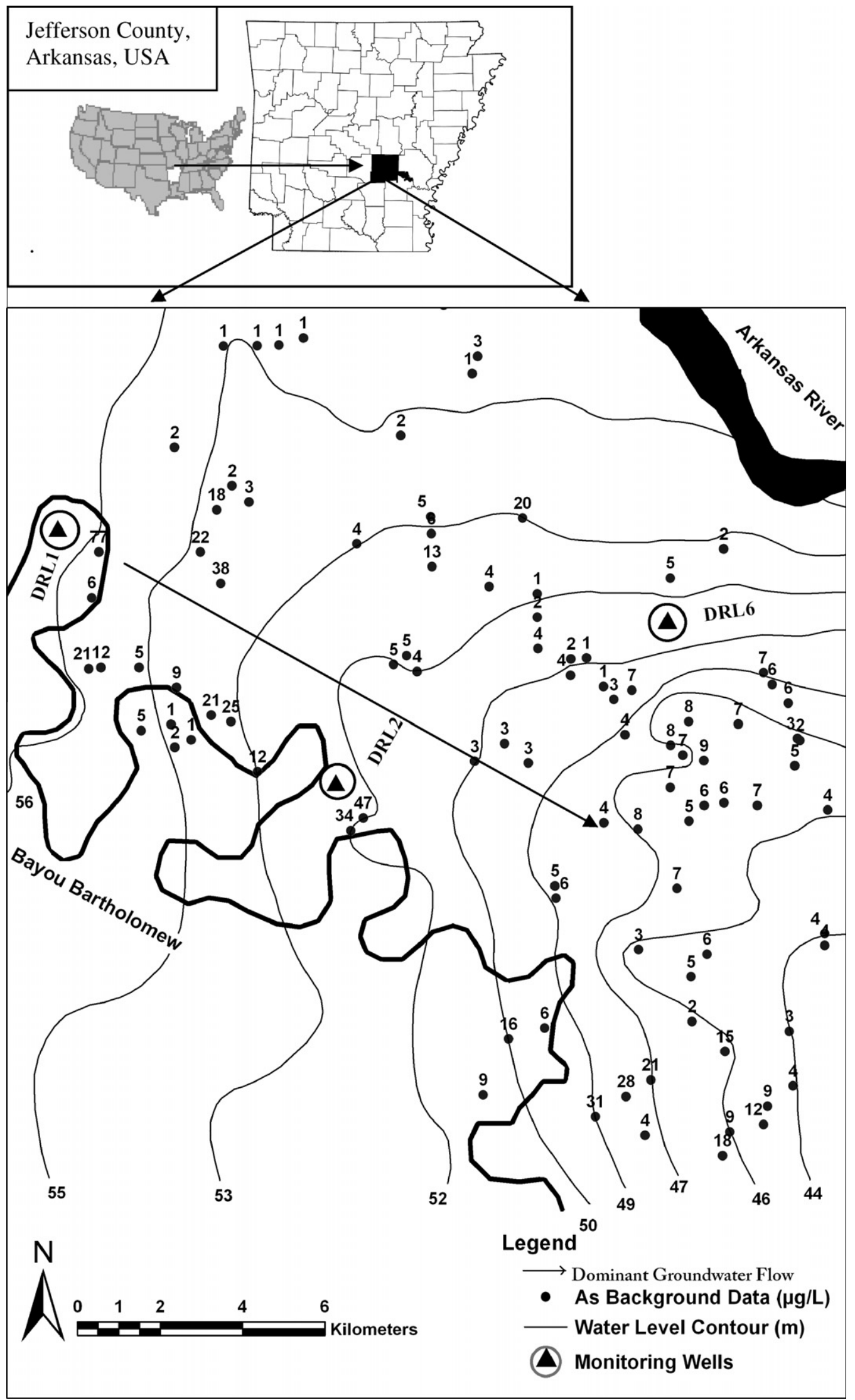

Fig. 1. Location of study area, monitoring well sites, water level map, and As background data in groundwater (Note: modified from Fig. 1 of Sharif et al. (2008a,b)). 


\subsection{Preparation of sediment samples and sequential extraction}

Sealed sections of the stored sediment samples were opened and sub-sampled in February 2006 for grain-size, porosity and geochemical analyses. Approximately $100 \mathrm{~g}$ of stored sediment cores were separated and dried below $40{ }^{\circ} \mathrm{C}$ in an oven. The sediment samples were crushed using a conventional porcelain pestle and mortar, and passed through a $1 \mathrm{~mm}$ screen. These screened sediment samples were used for a 5-step sequential extraction procedure (Tessier et al., 1979; Chao and Zhou, 1983) for major cations and trace metals, and As. Grain-size analysis of the fine fraction was conducted with little or no crushing on dried pre-screened samples by a micro-pipette method (Miller and Miller, 1987). Porosity was measured by weighing $50 \mathrm{~mL}$ hand-packed sediments in a graduated cylinder. Water was slowly added to the $50 \mathrm{~mL}$ mark and the sample shaken to remove air bubbles and saturate evenly with water. Gravimetric porosity $\left[\left(1-\left(\rho_{b} / \rho_{s}\right)\right]\right.$ was calculated by mean particle density $\left(\rho_{s}=\right.$ mass of solids/volume of solids) and dry bulk density ( $\rho_{b}=$ mass of dry solids/volume of dry solids). The 5-step sequential extraction procedure (Tessier et al., 1979; Chao and Zhou, 1983) was completed using 2 g dry-sieved sediment by the procedures listed below. Details of the 5-step sequential extraction procedure and results are presented in Sharif (2007) and Sharif et al. (2008a,b). The results of leachable fractions of As extracted by step 3 (Chao reagent) and step 5 (hot $\mathrm{HNO}_{3}$ ) were used in the simulation of SCMs as shown in Tables 1 and 2:

1. Exchangeable: $16 \mathrm{~mL}$ of $1 \mathrm{M} \mathrm{Na}$ acetate to $\mathrm{pH} 8.2$ for $1 \mathrm{~h}$.

2. Carbonates: $16 \mathrm{~mL}$ of $1 \mathrm{M} \mathrm{Na}$ acetate to $\mathrm{pH} 5$ for $4 \mathrm{~h}$.

3. Amorphous Fe and $\mathrm{Mn}$ oxides: $40 \mathrm{~mL}$ of $0.25 \mathrm{M} \mathrm{NH}_{2} \mathrm{OH}-\mathrm{HCl}$ in $0.25 \mathrm{M} \mathrm{HCl}$; heated to $50{ }^{\circ} \mathrm{C}$ for $30 \mathrm{~min}$.

4. Organic matter: $6 \mathrm{~mL}$ of $0.02 \mathrm{M} \mathrm{HNO}_{3}$ and $10 \mathrm{~mL}$ of $30 \% \mathrm{H}_{2} \mathrm{O}_{2}$ to $\mathrm{pH} 2$ with $\mathrm{HNO}_{3}$; heated to $85{ }^{\circ} \mathrm{C}$ for $2 \mathrm{~h}$, and later $6 \mathrm{~mL}$ of $30 \%$ $\mathrm{H}_{2} \mathrm{O}_{2}$ added and heated to $85^{\circ} \mathrm{C}$ for $3 \mathrm{~h}$.

5. Hot $\mathrm{HNO}_{3}$-leachable: $15 \mathrm{~mL} 7 \mathrm{M} \mathrm{HNO}_{3}$ for $2.5 \mathrm{~h}$ at $70{ }^{\circ} \mathrm{C}$ for the first $30 \mathrm{~min}$. and later at $100{ }^{\circ} \mathrm{C}$ for the next $2 \mathrm{~h}$.

The hot $\mathrm{HNO}_{3}$ extraction (Andersson et al., 1991) step in the sequential extraction procedures was used instead of the rigorous
$\mathrm{HF}-\mathrm{HClO}_{4}$ extraction described in the original method to represent the least environmentally-available As. A total of 60 sediment samples were extracted by using these extraction procedures. The extracted solutions were shipped to the Arkansas Department of Environmental Quality (ADEQ) laboratory, Little Rock, Arkansas, for analysis by inductively coupled plasma mass spectrometry (ICP-MS).

XRD and SEM analyses of sediments were conducted and are described in detail in Sharif (2007) and Sharif et al. (2008a,b). Detailed descriptions of the groundwater sampling, field monitoring, and laboratory analyses protocols and procedures are also included in the previously cited publications.

\subsection{Geochemical modeling}

The equilibrium SCM of PHREEQC (Parkhurst and Appelo, 1999) was used to predict the differences between sorbed As on HFO phases derived from sequentially extracted chemical data and modeled simulations. The DLM model of Dzombak and Morel (1990) was used to simulate surface complexation reactions. The model sorbents were selected as ferrihydrite and goethite. Ligand sorption was considered to occur at two sorption sites on ferrihydrite with a density of $0.005 \mathrm{~mol} / \mathrm{mol} \mathrm{Fe}$ for the strong site, and $0.2 \mathrm{~mol} / \mathrm{mol}$ Fe for the weak site (Dzombak and Morel, 1990). Ligand sorption for goethite was considered to occur at only one sorption site with a density of $0.00000384 \mathrm{~mol}$ sites $/ \mathrm{m}^{2}$ (Manning and Goldberg, 1996). The surface areas of ferrihydite and goethite were considered as $600 \mathrm{~m}^{2} / \mathrm{g}$ and $43.7 \mathrm{~m}^{2} / \mathrm{g}$, respectively (Dzombak and Morel, 1990; Manning and Goldberg, 1996). The surfacesite density of sorbent phases for individual sediment samples was calculated from: (1) the Fe concentrations in the sediment extracts generated using Chao reagent (Table 1) and hot $\mathrm{HNO}_{3}$ (Table 2) of Tessier's 5-step sequential extraction procedures, (2) the water-rock ratio from measured porosity, (3) assumed molecular weight, and (4) default surface-site density of sorbent phases (Dzombak and Morel, 1990; Manning and Goldberg, 1996). The WATEQ4F thermodynamic database of PHREEQC was used for surface reactions between As and ferrihydrite. The database was modified by adding silica sorption data on ferrihydrite from Swedlund

Table 1

Surface site densities of ferrihydrite and goethite based on chemical data extracted by Chao reagent. Site density is expressed as mol sites/mol Fe.

\begin{tabular}{|c|c|c|c|c|c|c|c|c|c|}
\hline Sr. no. & Sample ID & Lithology & $\begin{array}{l}\text { Depth, } \\
\text { m bgs }\end{array}$ & $\begin{array}{l}\text { Fe (Chao), } \\
\mathrm{mg} / \mathrm{kg}\end{array}$ & $\begin{array}{l}\text { kg Seds. } \\
\text { per kg } \mathrm{H}_{2} \mathrm{O}\end{array}$ & $\begin{array}{l}\text { Ferrihydrite, } \\
\mathrm{g} / \mathrm{kg} \mathrm{H}_{2} \mathrm{O}\end{array}$ & $\begin{array}{l}\text { Ferrihydrite } \\
\text { strong site }\end{array}$ & $\begin{array}{l}\text { Ferrihydrite } \\
\text { weak site }\end{array}$ & $\begin{array}{l}\text { Goethite } \\
\text { single site }\end{array}$ \\
\hline 1 & DRL12615 & Clayey silt & 6.1 & $9.2 \mathrm{E}+02$ & 8.00 & 11.67 & $6.57 \mathrm{E}-04$ & $2.63 \mathrm{E}-02$ & $1.96 \mathrm{E}-03$ \\
\hline 2 & DRL12617 & Sandy silt & 6.4 & $6.8 \mathrm{E}+02$ & 7.35 & 7.92 & $4.46 \mathrm{E}-04$ & $1.78 \mathrm{E}-02$ & $1.33 \mathrm{E}-03$ \\
\hline 3 & DRL12618 & Clayey silt & 7.0 & $8.3 E+02$ & 8.00 & 10.53 & $5.93 \mathrm{E}-04$ & $2.61 \mathrm{E}-02$ & $1.77 \mathrm{E}-03$ \\
\hline 4 & DRL12621 & Silty sand & 7.6 & $2.7 \mathrm{E}+02$ & 5.72 & 2.48 & $1.39 \mathrm{E}-04$ & $5.58 \mathrm{E}-03$ & $4.16 \mathrm{E}-04$ \\
\hline 5 & DRL12623-1 & Clayey silt & 8.5 & $6.2 \mathrm{E}+02$ & 8.00 & 7.85 & $4.42 \mathrm{E}-04$ & $1.77 \mathrm{E}-02$ & $1.32 \mathrm{E}-03$ \\
\hline 6 & DRL12624 & Clayey silt & 8.8 & $6.7 \mathrm{E}+02$ & 8.00 & 8.49 & $4.78 \mathrm{E}-04$ & $1.91 \mathrm{E}-02$ & $1.42 \mathrm{E}-03$ \\
\hline 7 & DRL12625-1 & Sand & 11.0 & $5.7 \mathrm{E}+02$ & 6.18 & 5.55 & $3.13 \mathrm{E}-04$ & $1.25 \mathrm{E}-02$ & $9.32 \mathrm{E}-04$ \\
\hline 8 & DRL12634 & Sand & 24.4 & $1.5 \mathrm{E}+02$ & 6.18 & 1.51 & $8.53 \mathrm{E}-05$ & $3.41 \mathrm{E}-03$ & $2.54 \mathrm{E}-04$ \\
\hline 9 & DRL12635 & Sand & 25.9 & $1.3 \mathrm{E}+02$ & 6.18 & 1.32 & $7.45 \mathrm{E}-05$ & $2.98 \mathrm{E}-03$ & $2.22 \mathrm{E}-04$ \\
\hline 10 & DRL12638-3 & Sand & 33.5 & $3.9 \mathrm{E}+02$ & 3.98 & 2.46 & $1.39 \mathrm{E}-04$ & $5.55 \mathrm{E}-03$ & $4.14 \mathrm{E}-04$ \\
\hline 11 & DRL12639-3 & Sand & 36.6 & $5.5 \mathrm{E}+02$ & 6.18 & 5.41 & $3.04 \mathrm{E}-04$ & $1.22 \mathrm{E}-02$ & $9.07 \mathrm{E}-04$ \\
\hline 12 & DRL12640 & Clay lens & 36.6 & $1.5 \mathrm{E}+03$ & 8.00 & 19.65 & $1.11 \mathrm{E}-03$ & $4.43 \mathrm{E}-02$ & $3.30 \mathrm{E}-03$ \\
\hline 13 & DRL22610 & Silty sand & 6.7 & $6.1 \mathrm{E}+02$ & 7.35 & 7.16 & $4.03 \mathrm{E}-04$ & $1.61 \mathrm{E}-02$ & $1.20 \mathrm{E}-03$ \\
\hline 14 & DRL22611 & Silt & 7.6 & $2.0 \mathrm{E}+03$ & 8.00 & 24.90 & $1.40 \mathrm{E}-03$ & $5.61 \mathrm{E}-02$ & $4.18 \mathrm{E}-03$ \\
\hline 15 & DRL22612 & Sand & 9.1 & $4.7 \mathrm{E}+02$ & 6.18 & 4.62 & $2.60 \mathrm{E}-04$ & $1.04 \mathrm{E}-02$ & $7.75 \mathrm{E}-04$ \\
\hline 16 & DRL22613-2 & Sand & 10.7 & $1.6 \mathrm{E}+03$ & 6.18 & 15.51 & $8.73 E-04$ & $3.49 \mathrm{E}-02$ & $2.60 \mathrm{E}-03$ \\
\hline 17 & DRL22618-2 & Sand & 18.3 & $6.4 \mathrm{E}+01$ & 6.18 & 0.62 & $3.52 \mathrm{E}-05$ & $1.41 \mathrm{E}-03$ & $1.05 \mathrm{E}-04$ \\
\hline 18 & DRL22620-2 & Sand & 21.3 & $1.2 \mathrm{E}+02$ & 6.18 & 1.17 & $6.59 \mathrm{E}-05$ & $2.64 \mathrm{E}-03$ & $1.96 \mathrm{E}-04$ \\
\hline 19 & DRL22623 & Sand & 30.5 & $1.9 \mathrm{E}+02$ & 6.18 & 1.86 & $1.05 \mathrm{E}-04$ & $4.19 \mathrm{E}-03$ & $3.12 \mathrm{E}-04$ \\
\hline 20 & DRL62615 & Clayey sand & 8.5 & $1.4 \mathrm{E}+03$ & 7.95 & 17.10 & $9.63 \mathrm{E}-04$ & $3.85 \mathrm{E}-02$ & $2.87 \mathrm{E}-03$ \\
\hline 21 & DRL62616 & Silty sand & 8.8 & $1.5 \mathrm{E}+03$ & 7.35 & 17.15 & $9.66 \mathrm{E}-04$ & $3.86 \mathrm{E}-02$ & $2.88 \mathrm{E}-03$ \\
\hline 22 & DRL62618 & Silty sand & 10.1 & $1.0 \mathrm{E}+04$ & 7.35 & 117.16 & $6.60 \mathrm{E}-03$ & $2.64 \mathrm{E}-01$ & $1.97 \mathrm{E}-02$ \\
\hline 23 & DRL62619 & Sand & 10.2 & $7.1 \mathrm{E}+02$ & 6.18 & 6.93 & $3.90 \mathrm{E}-04$ & $1.56 \mathrm{E}-02$ & $1.16 \mathrm{E}-03$ \\
\hline 24 & DRL62625 & Sand & 18.3 & $1.8 \mathrm{E}+03$ & 6.18 & 18.02 & $1.01 \mathrm{E}-03$ & $4.06 \mathrm{E}-02$ & $3.02 \mathrm{E}-03$ \\
\hline 25 & DRL62626 & Sand & 21.3 & $1.3 \mathrm{E}+03$ & 6.18 & 12.83 & $7.22 \mathrm{E}-04$ & $2.89 \mathrm{E}-02$ & $2.15 \mathrm{E}-03$ \\
\hline 26 & DRL62628 & Sand & 24.4 & $8.4 \mathrm{E}+02$ & 6.18 & 8.26 & $4.65 \mathrm{E}-04$ & $1.86 \mathrm{E}-02$ & $1.39 \mathrm{E}-03$ \\
\hline
\end{tabular}


Table 2

Surface site densities of ferrihydrite and goethite based on chemical data extracted by hot $\mathrm{HNO}_{3}$. Site density is expressed as mol sites/mol Fe.

\begin{tabular}{|c|c|c|c|c|c|c|c|c|c|}
\hline Sr. no. & Sample ID & Lithology & $\begin{array}{l}\text { Depth, } \\
\text { m }\end{array}$ & $\begin{array}{l}\mathrm{Fe}\left(\mathrm{HNO}_{3}\right), \\
\mathrm{mg} / \mathrm{kg} \text { seds }\end{array}$ & $\begin{array}{l}\text { kg Seds. } \\
\text { per kg } \mathrm{H}_{2} \mathrm{O}\end{array}$ & $\begin{array}{l}\text { Ferrihydrite, } \\
\mathrm{g} / \mathrm{kg} \mathrm{H}_{2} \mathrm{O}\end{array}$ & $\begin{array}{l}\text { Ferrihydrite } \\
\text { strong site }\end{array}$ & $\begin{array}{l}\text { Ferrihydrite } \\
\text { weak site }\end{array}$ & $\begin{array}{l}\text { Goethite } \\
\text { single site }\end{array}$ \\
\hline 1 & DRL12615 & Clayey silt & 6.1 & 9100 & 8.00 & 115.75 & $6.5 \mathrm{E}-03$ & $2.6 \mathrm{E}-01$ & $1.9 \mathrm{E}-02$ \\
\hline 2 & DRL12617 & Sandy silt & 6.4 & 7375 & 7.35 & 86.19 & $4.9 \mathrm{E}-03$ & $1.9 \mathrm{E}-01$ & $1.4 \mathrm{E}-02$ \\
\hline 3 & DRL12618 & Clayey silt & 7.0 & 15,300 & 8.00 & 194.62 & $1.1 \mathrm{E}-02$ & $4.4 \mathrm{E}-01$ & $3.3 \mathrm{E}-02$ \\
\hline 4 & DRL12621 & Silty sand & 7.6 & 4700 & 5.72 & 42.72 & $2.4 \mathrm{E}-03$ & $9.6 \mathrm{E}-02$ & $7.2 \mathrm{E}-03$ \\
\hline 5 & DRL12623-1 & Clayey silt & 8.5 & 11,050 & 8.00 & 140.56 & $7.9 \mathrm{E}-03$ & $3.2 \mathrm{E}-01$ & $2.4 \mathrm{E}-02$ \\
\hline 6 & DRL12624 & Clayey silt & 8.8 & 12,825 & 8.00 & 163.13 & $9.2 \mathrm{E}-03$ & $3.7 \mathrm{E}-01$ & $2.7 \mathrm{E}-02$ \\
\hline 7 & DRL12625-1 & Sand & 11.0 & 3475 & 6.18 & 34.16 & $1.9 \mathrm{E}-03$ & $7.7 \mathrm{E}-02$ & $5.7 \mathrm{E}-03$ \\
\hline 8 & DRL12634 & Sand & 24.4 & 518 & 6.18 & 5.09 & $2.9 \mathrm{E}-04$ & $1.1 \mathrm{E}-02$ & $8.5 \mathrm{E}-04$ \\
\hline 9 & DRL12635 & Sand & 25.9 & 1080 & 6.18 & 10.62 & $6.0 \mathrm{E}-04$ & $2.4 \mathrm{E}-02$ & $1.8 \mathrm{E}-03$ \\
\hline 10 & DRL12638-3 & Sand & 33.5 & 1593 & 3.98 & 10.06 & $5.7 \mathrm{E}-04$ & $2.3 \mathrm{E}-02$ & $1.7 \mathrm{E}-03$ \\
\hline 11 & DRL12639-3 & Sand & 36.6 & 4700 & 6.18 & 46.21 & $2.6 \mathrm{E}-03$ & $1.0 \mathrm{E}-01$ & $7.8 \mathrm{E}-03$ \\
\hline 12 & DRL12640 & Clay lens & 36.6 & 7600 & 8.00 & 96.67 & $5.4 \mathrm{E}-03$ & $2.2 \mathrm{E}-01$ & $1.6 \mathrm{E}-02$ \\
\hline 13 & DRL22610 & Silty sand & 6.7 & 3550 & 7.35 & 63.97 & $3.6 \mathrm{E}-03$ & $1.4 \mathrm{E}-01$ & $1.1 \mathrm{E}-02$ \\
\hline 14 & DRL22611 & Silt & 7.6 & 12,775 & 8.00 & 161.48 & $9.1 \mathrm{E}-03$ & $3.6 \mathrm{E}-01$ & $2.7 \mathrm{E}-02$ \\
\hline 15 & DRL22612 & Sand & 9.1 & 1943 & 6.18 & 24.55 & $1.4 \mathrm{E}-03$ & $5.5 \mathrm{E}-02$ & $4.1 \mathrm{E}-03$ \\
\hline 16 & DRL22613-2 & Sand & 10.7 & 17,300 & 6.18 & 218.68 & $1.2 \mathrm{E}-02$ & $4.9 \mathrm{E}-01$ & $3.7 \mathrm{E}-02$ \\
\hline 17 & DRL22618-2 & Sand & 18.3 & 618 & 6.18 & 6.07 & $3.4 \mathrm{E}-04$ & $1.4 \mathrm{E}-02$ & $1.0 \mathrm{E}-03$ \\
\hline 18 & DRL22620-2 & Sand & 21.3 & 1208 & 6.18 & 11.87 & $6.7 \mathrm{E}-04$ & $2.7 \mathrm{E}-02$ & $2.0 \mathrm{E}-03$ \\
\hline 19 & DRL22623 & Sand & 30.5 & 868 & 6.18 & 8.53 & $4.8 \mathrm{E}-04$ & $1.9 \mathrm{E}-02$ & $1.4 \mathrm{E}-03$ \\
\hline 20 & DRL62615 & Clayey sand & 8.5 & 8450 & 7.95 & 106.81 & $6.0 \mathrm{E}-03$ & $2.4 \mathrm{E}-01$ & $1.8 \mathrm{E}-02$ \\
\hline 21 & DRL62616 & Silty sand & 8.8 & 4175 & 7.35 & 48.79 & $2.7 \mathrm{E}-03$ & $1.1 \mathrm{E}-01$ & $8.2 \mathrm{E}-03$ \\
\hline 22 & DRL62618 & Silty sand & 10.1 & 16,950 & 7.35 & 198.09 & $1.1 \mathrm{E}-02$ & $4.5 \mathrm{E}-01$ & $3.3 \mathrm{E}-02$ \\
\hline 23 & DRL62619 & Sand & 10.2 & 1155 & 6.18 & 11.36 & $6.4 \mathrm{E}-04$ & $2.6 \mathrm{E}-02$ & $1.9 \mathrm{E}-03$ \\
\hline 24 & DRL62625 & Sand & 18.3 & 2625 & 6.18 & 25.81 & $1.5 \mathrm{E}-03$ & $5.8 \mathrm{E}-02$ & $4.3 \mathrm{E}-03$ \\
\hline 25 & DRL62626 & Sand & 21.3 & 3175 & 6.18 & 31.22 & $1.8 \mathrm{E}-03$ & $7.0 \mathrm{E}-02$ & $5.2 \mathrm{E}-03$ \\
\hline 26 & DRL62628 & Sand & 24.4 & 2078 & 6.18 & 20.42 & $1.2 \mathrm{E}-03$ & $4.6 \mathrm{E}-02$ & $3.4 \mathrm{E}-03$ \\
\hline
\end{tabular}

Table 3

Important sorption reactions with surface constants of As with ferrihydrite and goethite that were added to WATEQ4F thermodynamic database in PHREEQC.

\begin{tabular}{|c|c|c|}
\hline Surface reaction & $\log K_{i n t}$ & References \\
\hline \multicolumn{3}{|l|}{ Ferrihydrite } \\
\hline $\mathrm{Hfo} \_\mathrm{OH}+\mathrm{H}_{4} \mathrm{SiO}_{4}=\mathrm{Hfo} \_\mathrm{H}_{3} \mathrm{SiO}_{4}+\mathrm{H}_{2} \mathrm{O}$ & 4.28 & Swedlund and Webster (1999) \\
\hline $\mathrm{Hfo} \_\mathrm{OH}+\mathrm{H}_{4} \mathrm{SiO}_{4}=\mathrm{Hfo} \_\mathrm{H}_{2} \mathrm{SiO}_{4}^{-}+\mathrm{H}_{2} \mathrm{O}+\mathrm{H}^{+}$ & -3.22 & Swedlund and Webster (1999) \\
\hline $\mathrm{Hfo} \_\mathrm{OH}+\mathrm{H}_{4} \mathrm{SiO}_{4}=\mathrm{Hfo} \_\mathrm{H}_{2} \mathrm{SiO}_{4}^{-}+\mathrm{H}_{2} \mathrm{O}+2 \mathrm{H}^{+}$ & -11.69 & Swedlund and Webster (1999) \\
\hline \multicolumn{3}{|l|}{ Goethite } \\
\hline Goe_OH $+\mathrm{H}^{+}=\mathrm{Goe}_{-} \mathrm{OH}^{2+}$ & 7.52 & Dixit and Hering (2003) \\
\hline 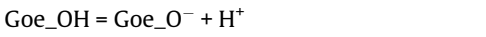 & -10.6 & Dixit and Hering (2003) \\
\hline Goe_OH $+\mathrm{H}_{3} \mathrm{PO}_{4}=$ Goe_ $\mathrm{H}_{2} \mathrm{PO}_{4}+\mathrm{H}_{2} \mathrm{O}$ & 8.05 & Sigg (1979) \\
\hline Goe_OH $+\mathrm{H}_{3} \mathrm{PO}_{4}=\mathrm{Goe} \_\mathrm{HPO}^{4-}+\mathrm{H}^{2} \mathrm{O}+\mathrm{H}^{+}$ & 3.40 & Sigg (1979) \\
\hline Goe_OH $+\mathrm{H}_{3} \mathrm{PO}_{4}=\mathrm{Goe}_{-} \mathrm{PO} 4^{-2}+\mathrm{H}_{2} \mathrm{O}+2 \mathrm{H}^{+}$ & -2.20 & Sigg (1979) \\
\hline $\mathrm{Goe} \_\mathrm{OH}+\mathrm{AsO}_{4}^{-3}+3 \mathrm{H}^{+}=\mathrm{Goe}_{-} \mathrm{H}_{2} \mathrm{AsO}_{4}+\mathrm{H}_{2} \mathrm{O}$ & 30.94 & Dixit and Hering (2003) \\
\hline $\mathrm{Goe} \_\mathrm{OH}+\mathrm{AsO}_{4}^{-3}+2 \mathrm{H}^{+}=\mathrm{Goe}_{-} \mathrm{HAsO}^{4-}+\mathrm{H}_{2} \mathrm{O}$ & 26.75 & Dixit and Hering (2003) \\
\hline $\mathrm{Goe} \_\mathrm{OH}+\mathrm{AsO}_{4}^{-3}+\mathrm{H}^{+}=\mathrm{Goe}_{-} \mathrm{AsO}_{4}^{-2}+\mathrm{H}_{2} \mathrm{O}$ & 20.16 & Dixit and Hering (2003) \\
\hline $\mathrm{Goe} \_\mathrm{OH}+\mathrm{AsO}_{3}^{-3}+3 \mathrm{H}^{+}=\mathrm{Goe}_{-} \mathrm{H}_{2} \mathrm{AsO}_{3}+\mathrm{H}_{2} \mathrm{O}$ & 39.87 & Dixit and Hering (2003) \\
\hline $\mathrm{Goe} \_\mathrm{OH}+\mathrm{AsO}_{3}^{-3}+2 \mathrm{H}^{+}=\mathrm{Goe}_{-} \mathrm{HAsO}_{3}^{-}+\mathrm{H}_{2} \mathrm{O}$ & 32.34 & Dixit and Hering (2003) \\
\hline $\mathrm{Goe} \_\mathrm{OH}+\mathrm{H}_{4} \mathrm{SiO}_{4}^{-3}=$ Goe $\_\mathrm{H}_{3} \mathrm{SiO}_{4}+\mathrm{H}_{2} \mathrm{O}$ & 4.35 & Sigg (1979) \\
\hline $\mathrm{Goe} \_\mathrm{OH}+\mathrm{H}_{4} \mathrm{SiO}_{4}=\mathrm{Goe}_{-} \mathrm{H}_{2} \mathrm{SiO}_{4}^{-}+\mathrm{H}_{2} \mathrm{O}+\mathrm{H}^{+}$ & -3.04 & Sigg (1979) \\
\hline $\mathrm{Goe} \_\mathrm{OH}+2 \mathrm{H}^{+}+\mathrm{CO}_{3}^{-2}=\mathrm{Goe} \_\mathrm{HCO}_{3}+\mathrm{H}_{2} \mathrm{O}$ & 20.78 & Van Geen et al. (1994) \\
\hline $\mathrm{Goe} \_\mathrm{OH}+\mathrm{H}^{+}+\mathrm{CO}_{3}^{-2}=\mathrm{Goe}_{-} \mathrm{CO}_{3}^{-}+\mathrm{H}_{2} \mathrm{O}$ & 12.71 & Van Geen et al. (1994) \\
\hline $\mathrm{Goe} \_\mathrm{OH}+\mathrm{CO}_{3}^{-2}=\mathrm{Goe} \_\mathrm{OHCO}-{ }_{3}^{-2}$ & 3.56 & Appelo et al. (2002) \\
\hline Goe_OH $+\mathrm{F}^{-}=\mathrm{Goe} \_\mathrm{F}+\mathrm{OH}^{-}$ & -4.54 & Sigg (1979) \\
\hline $\mathrm{Goe} \_\mathrm{OH}+\mathrm{SO}_{4}^{2-}+2 \mathrm{H}^{+}=\mathrm{Goe}_{-} \mathrm{HSO}_{4}+\mathrm{H}_{2} \mathrm{O}$ & 13.61 & Ali and Dzombak (1996) \\
\hline $\mathrm{Goe} \_\mathrm{OH}+\mathrm{SO}_{4}^{2-}+\mathrm{H}^{+}=\mathrm{Goe} \_\mathrm{SO}_{4}^{-}+\mathrm{H}_{2} \mathrm{O}$ & 8.19 & Ali and Dzombak (1996) \\
\hline $\mathrm{Goe} \_\mathrm{OH}+\mathrm{SO}_{4}^{2-}=\mathrm{Goe}_{-} \mathrm{OSO}_{4}^{3-}+\mathrm{H}^{+}$ & -6.26 & Ali and Dzombak (1996) \\
\hline $\mathrm{Goe} \_\mathrm{OH}+\mathrm{SO}_{4}^{2-}+\mathrm{Cu}^{2+}=\mathrm{Goe} \_\mathrm{OHCuSO} 4$ & 9.46 & Ali and Dzombak (1996) \\
\hline $\mathrm{Goe} \_\mathrm{OH}+\mathrm{Zn}^{2+}+\mathrm{H}_{2} \mathrm{O}=\mathrm{Goe} \_\mathrm{OZn}(\mathrm{OH})_{2}^{-}+3 \mathrm{H}^{+}$ & -18.34 & Palmqvist et al. (1999) \\
\hline $\mathrm{Goe} \_\mathrm{OH}+\mathrm{Zn}^{2+}=\mathrm{Goe} \_\mathrm{OZn}{ }^{+}+\mathrm{H}^{+}$ & -2.14 & Palmqvist et al. (1999) \\
\hline Goe_OH $+\mathrm{Fe}^{2+}+\mathrm{H}_{2} \mathrm{O}=$ Goe_OFeOH ${ }^{+}+2 \mathrm{H}^{+}$ & -10.95 & Dixit and Hering (2006) \\
\hline Goe_OH $+\mathrm{Fe}^{2+}=$ Goe_OFe ${ }^{+}+\mathrm{H}^{+}$ & -0.60 & Dixit and Hering (2006) \\
\hline $\mathrm{Goe} \_\mathrm{OH}+\mathrm{Cu}^{2+}=\mathrm{Goe}_{-} \mathrm{OCu}^{+}+\mathrm{H}^{+}$ & 2.93 & Weirich (2000) \\
\hline $\mathrm{Goe} \_\mathrm{OH}+\mathrm{Ni}^{2+}=\mathrm{Goe} \_\mathrm{ONi}^{+}+\mathrm{H}^{+}$ & 0.46 & Weirich (2000) \\
\hline Goe $\_$OH $+\mathrm{H}_{3} \mathrm{BO}_{3}=$ Goe $\_\mathrm{H}_{2} \mathrm{BO}_{3}^{+}+\mathrm{H}_{2} \mathrm{O}$ & 5.52 & Goldberg and Glaubig (1985) \\
\hline Goe $\_\mathrm{OH}+\mathrm{Ca}^{2+}=\mathrm{Goe}_{-} \mathrm{OCa}^{+}+\mathrm{H}^{+}$ & -7.18 & Ali and Dzombak (1996) \\
\hline Goe_OH $+\mathrm{Mg}^{2+}=$ Goe_OMg ${ }^{+}+\mathrm{H}^{+}$ & -5.94 & Sigg (1979) \\
\hline
\end{tabular}


and Webster (1999), which is internally consistent with the sorption database of Dzombak and Morel (1990). The surface reactions for goethite were taken from the RES3T (Rossendorf Expert System for Surface and Sorption Thermodynamics) thermodynamic sorption database (Brendler et al., 2002), which includes sorption constants for the DLM model summarized from different sources. The sorption database of goethite was normalized by converting the reference site density to 2.31 sites $/ \mathrm{nm}^{2}$ for all surface reactions to get internal consistency, following the procedures of Kulik (2002). Important sorption reaction constants that were added to the WATEQ4F thermodynamic database of PHREEQC are given in Table 3 . The model was tested on 26 selected sediment samples collected along the depth profile of six boreholes (DRL1S, DRL1D, DRL2S, DRL2D, DRL6S and DRL6D). The data for chemical analyses of groundwater from corresponding monitoring wells were used in the respective model input (Sharif, 2007; Sharif et al., 2008a,b). Groundwater chemistry of the shallow well was used in the model input for sediments up to a depth of $10.6 \mathrm{~m}$. Groundwater chemistry of the deep well was used in the model input for sediments collected from 18 to $36.6 \mathrm{~m}$.

\section{Results}

Detailed results for sediment geochemistry, groundwater chemistry, XRD and SEM analyses, and interpretation of these analyses in the light of redox conditions and As mobilization mechanisms are described by Sharif (2007) and Sharif et al. (2008a,b). The following section summarizes the As mobilization mechanisms reported elsewhere (Sharif, 2007; Sharif et al., 2008a,b) and the results of the SCM approach.

\subsection{As mobilization mechanisms}

Arsenic is positively correlated to Fe extracted by Chao reagent $(r=0.83)$ and $\mathrm{HNO}_{3}(r=0.85)$. Iron speciation data in sediments vary with depth in the aquifer (Sharif et al., 2008b). Increasing depth has a positive relationship $(r=0.56)$ with the solid phase $\mathrm{Fe}(\mathrm{II}) / \mathrm{Fe}$ ratio (the ratio of Fe concentration in the extracts of Chao reagent and hot $\mathrm{HNO}_{3}$ ), but it has a negative relationship ( $r=$ -0.45 ) with As extracted by Chao reagent. The solid phase Fe(II)/ Fe ratio is positively correlated $(r=0.76)$ to As extracted by Chao reagent. Although the solid phase $\mathrm{Fe}(\mathrm{II}) / \mathrm{Fe}$ ratio increases with depth, the amount of reducible Fe (HFO), as well as its complexed As, decreases with depth. One cannot ignore the possibility of historic flushing of significant dissolved As derived from reductive dissolution of HFO by advective transport, coupled with less sorptive capacity of HFO with later development of crystallinity at depth. Interpretation of geochemical data reveals that both amorphous and crystalline Fe oxide phases in the shallow aquifer are under relatively oxidizing conditions, and represent a potential sink for As. Amorphous and crystalline Fe oxide phases in the deep aquifer are under a reducing environment, favoring reductive dissolution of HFO and concomitant release of As into groundwater. The sharp depletion of $\mathrm{SO}_{4}^{2-}$ concentrations in groundwater from shallow (DRL1S) to deep (DRL1D) monitoring wells may be the result of simultaneous reduction of $\mathrm{HFO}$ and $\mathrm{SO}_{4}^{2-}$ with co-precipitation of dissolved As into sulfides. This is supported by a mutual exclusivity of As with $\mathrm{SO}_{4}^{2-}$ from 118 well-water analyses in the Bayou Bartholomew watershed (Kresse and Fazio, 2002).

\subsection{Surface complexation modeling}

The DLM option of the SCM in the geochemical code PHREEQC was implemented using ferrihydrite and goethite as the sorbents. The model was run using As data for sediments extracted by Chao reagent (As attached to amorphous Fe and Mn oxides and oxyhydroxides) and hot $\mathrm{HNO}_{3}$ (As attached to crystalline Fe and other metal oxides). The model results are satisfactory for DRL1S, where the redox condition is relatively oxic or mildly suboxic. However, for the deep alluvial aquifer (21-36.6 m) where the redox condition is suboxic to anoxic, the model results are unsatisfactory. The model shows a contrasting variation between modeled and extracted As in the sediments along the depth profile. Primarily, the model output is very sensitive to the sediment type and the extraction method used, which controls the surface-site density and sorption intensity. The model output is very sensitive to groundwater composition, which controls competitive sorption of

Table 4

Model results using As extracted by Chao reagent.

\begin{tabular}{|c|c|c|c|c|c|}
\hline \multirow[t]{2}{*}{ Sample ID } & \multirow[t]{2}{*}{ Lithology } & \multirow[t]{2}{*}{ Depth, m bgs ${ }^{a}$} & \multirow{2}{*}{$\frac{\text { Extracted } \mathrm{As}}{\text { Chao reagent }(\mathrm{mg} / \mathrm{kg})}$} & \multirow{2}{*}{$\begin{array}{l}\text { Model } \\
\text { Derived As (mg/kg) }\end{array}$} & \multirow{2}{*}{$\begin{array}{l}\text { Difference (\%) between } \\
\text { Modeled and extracted As (Chao reagent) }\end{array}$} \\
\hline & & & & & \\
\hline DRL12615 & Clayey silt & 6.1 & 0.68 & 0.34 & 49 \\
\hline DRL12617 & Sandy silt & 6.4 & 0.49 & 0.26 & 47 \\
\hline DRL12618 & Clayey silt & 7.0 & 0.57 & 0.32 & 44 \\
\hline DRL12621 & Silty sand & 7.6 & 0.23 & 0.10 & 55 \\
\hline DRL12623-1 & Clayey silt & 8.5 & 0.43 & 0.24 & 45 \\
\hline DRL12624 & clayey silt & 8.8 & 0.52 & 0.25 & 52 \\
\hline DRL12625-1 & Sand & 10.2 & 0.24 & 0.22 & 8 \\
\hline DRL12634 & Sand & 24.4 & 0.04 & 0.90 & -2149 \\
\hline DRL12635 & Sand & 25.9 & 0.21 & 0.79 & -286 \\
\hline DRL12638-3 & Sand & 33.5 & 0.14 & 1.72 & -1167 \\
\hline DRL12639-3 & Sand & 36.6 & 0.17 & 3.17 & -1767 \\
\hline DRL12640 & Clay lens & 36.6 & 1.10 & 8.99 & -717 \\
\hline DRL22610 & Silty sand & 6.7 & 0.22 & 1.42 & -548 \\
\hline DRL22611 & Silt & 7.6 & 0.56 & 4.71 & -737 \\
\hline DRL22612 & Sand & 9.1 & 0.12 & 1.63 & -1258 \\
\hline DRL22613-2 & Sand & 10.6 & 0.65 & 5.48 & -746 \\
\hline DRL22618-2 & Sand & 18.3 & 0.12 & 0.52 & -333 \\
\hline DRL22620-2 & Sand & 21.3 & 0.37 & 0.97 & -162 \\
\hline DRL22623 & Sand & 30.5 & 0.12 & 1.55 & -1192 \\
\hline DRL62615 & Clayey sand & 8.5 & 0.33 & 8.42 & -2491 \\
\hline DRL62616 & Silty sand & 8.8 & 0.21 & 11.72 & -5501 \\
\hline DRL62618 & Silty sand & 10.1 & 5.10 & 78.5 & -1439 \\
\hline DRL62619 & Sand & 10.2 & 2.02 & 4.43 & -119 \\
\hline
\end{tabular}

\footnotetext{
a bgs: Below ground surface.
} 
Table 5

Model results using As extracted by hot $\mathrm{HNO}$.

\begin{tabular}{|c|c|c|c|c|c|}
\hline \multirow[t]{2}{*}{ Sample ID } & \multirow[t]{2}{*}{ Lithology } & \multirow[t]{2}{*}{ Depth, m bgs ${ }^{*}$} & \multirow{2}{*}{$\frac{\text { Extracted As }}{\mathrm{HNO}_{3}(\mathrm{mg} / \mathrm{kg})}$} & \multirow{2}{*}{$\begin{array}{l}\text { Model } \\
\text { Derived As }(\mathrm{mg} / \mathrm{kg})\end{array}$} & \multirow{2}{*}{$\begin{array}{l}\text { Difference (\%) between } \\
\text { Modeled and extracted } \mathrm{As}\left(\mathrm{HNO}_{3}\right)\end{array}$} \\
\hline & & & & & \\
\hline DRL12615 & Clayey silt & 6.1 & 1.74 & 0.8 & 53 \\
\hline DRL12617 & Sandy silt & 6.4 & 1.41 & 0.7 & 54 \\
\hline DRL12618 & Clayey silt & 7.0 & 2.88 & 1.4 & 51 \\
\hline DRL12621 & Silty sand & 7.6 & 0.79 & 0.4 & 45 \\
\hline DRL12623-1 & Clayey silt & 8.5 & 1.29 & 1.0 & 21 \\
\hline DRL12624 & Clayey silt & 8.8 & 1.62 & 1.2 & 29 \\
\hline DRL12625-1 & Sand & 10.2 & 0.23 & 0.3 & -37 \\
\hline DRL12634 & Sand & 24.4 & 0.14 & 0.4 & -165 \\
\hline DRL12635 & Sand & 25.9 & 0.31 & 0.8 & -158 \\
\hline DRL12638-3 & Sand & 33.5 & 0.36 & 1.1 & -216 \\
\hline DRL12639-3 & Sand & 36.6 & 1.15 & 3.4 & -196 \\
\hline DRL12640 & Clay lens & 36.6 & 3.45 & 5.4 & -57 \\
\hline DRL22610 & Silty sand & 6.7 & 0.42 & 9.39 & -2122 \\
\hline DRL22611 & Silt & 7.6 & 1.72 & 21.18 & -1133 \\
\hline DRL22612 & Sand & 9.1 & 0.27 & 4.16 & -1470 \\
\hline DRL22613-2 & Sand & 10.6 & 3.60 & 37.58 & -944 \\
\hline DRL22618-2 & Sand & 18.3 & 0.14 & 1.56 & -1056 \\
\hline DRL22620-2 & Sand & 21.3 & 1.00 & 3.05 & -204 \\
\hline DRL22623 & Sand & 30.5 & 0.20 & 2.14 & -959 \\
\hline DRL62615 & Clayey sand & 8.5 & 0.86 & 33.88 & -3828 \\
\hline DRL62616 & Silty sand & 8.8 & 0.46 & 25.67 & -5480 \\
\hline DRL62618 & Silty sand & 10.1 & 22.8 & 66.34 & -191 \\
\hline DRL62619 & Sand & 10.2 & 2.40 & 4.6 & -92 \\
\hline
\end{tabular}

bgs: Below ground surface.

As with other ions (e.g. $\mathrm{HCO}_{3}^{-}, \mathrm{PO}_{4}^{3-}, \mathrm{H}_{4} \mathrm{SiO}_{4}^{0}$ and $\mathrm{Fe}^{2+}$ ) on reactive surface sites of sorbents.

For DRL1S (0-10 m), the model results (Tables 4 and 5) using ferrihydrite and goethite under-predicted $(<50 \%)$ the extracted As concentration in sediments. Sediments from all other boreholes except DRL1S using ferrihydrite and goethite highly over-predicted (up to 56 times) the extracted As concentration in sediments.

\section{Discussion}

Selection of the proper HFO phases is one of the most important factors influencing the model fit, as each phase has a different specific surface area and sorption capacity. The ferrihydrite used in the simulation has a surface area and surface-site density over 13 times greater than that of goethite. There are various lines of evidence for the presence of both ferrihydrite and goethite in the sediments of the study area. Field evidence for the presence of ferrihydrite, such as fine Fe oxide staining or coating on sand grains, is readily observable. These hydrous Fe oxide coatings are easily observable under SEM. Goethite was detected by XRD in all the sediment samples. Magnetically separated minerals show both crystalline and amorphous morphologies under SEM. Saturation index calculations, and the results of inverse modeling, show that groundwater is undersaturated with respect to goethite and ferrihydrite, and these are dissolving along the flow path in the study area (Sharif et al., 2008a). There is no direct quantitative evidence for the presence of selected sorbent phases in the sediments. The amount of ferrihydrite and goethite was quantified from partial and operationally defined wet chemical extractions. Concentrations of $\mathrm{Fe}$ in sediments extracted by Chao reagent and hot $\mathrm{HNO}_{3}$ extraction steps were used to back-calculate the amount of ferrihydrite and goethite using the molar ratios between ferrihydrite/Fe and goethite/Fe as 1.59. So, there are uncertainties in the selection and calculation of concentrations of the proper sorbent phases in the sediments used in the SCM.

Surface area and surface-site densities for selective sorbent phases are two important input parameters in the SCM simulation. Small variation in the surface-site densities of sorbents may pro- duce a difference of several orders of magnitude in the modeled concentrations of As in sediments. The best available approach is to use the default surface parameters found in published papers (Dzombak and Morel, 1990; Dixit and Hering, 2003, 2006; Manning and Goldberg, 1996) as a starting point to incorporate these parameters into the model. Ambiguity and inconsistency exist in the selection of these two important surface parameters, which increases the level of uncertainty in the application of SCMs to predict As occurrence in sediments.

The model output is very sensitive to the concentration of As and other competitive ions in groundwater. The model showed that $\mathrm{HCO}_{3}^{-}, \mathrm{H}_{4} \mathrm{SiO}_{4}, \mathrm{CO}_{3}^{2-}, \mathrm{PO}_{4}^{3-}$ and $\mathrm{Fe}^{2+}$ occupied more than $98 \%$ of reactive surface sites of HFO; so, the concentrations of these ionic species in groundwater and their respective stability constants play an important role in regulating the complexation of As on HFO surfaces. PHREEQC lacks surface reactions between As and $\mathrm{H}_{4} \mathrm{SiO}_{4}$, which produces a high level of uncertainty in the prediction capacity of the model as it highly overpredicts modeled As. Surface reactions between As and $\mathrm{H}_{4} \mathrm{SiO}_{4}$ were incorporated into the model to improve the prediction capacity of the model (Swedlund and Webster, 1999). There are some inconsistencies in the values of intrinsic surface constants in the surface reactions of As related to $\mathrm{HCO}_{3}^{-}, \mathrm{H}_{4} \mathrm{SiO}_{4}, \mathrm{CO}_{3}^{2-}, \mathrm{PO}_{4}^{3-}$, and other ions in different published papers (Dzombak and Morel, 1990; Swedlund and Webster, 1999; Dixit and Hering, 2003, 2006; Wilkie and Hering, 1996). Very small variations of intrinsic surface constants for $\mathrm{HCO}_{3}^{-}$, $\mathrm{H}_{4} \mathrm{SiO}_{4}, \mathrm{CO}_{3}^{2-}$ and $\mathrm{PO}_{4}^{3-}$ produces a large difference in the model output; so, appropriate selection of intrinsic surface constants related to $\mathrm{HCO}_{3}^{-}, \mathrm{H}_{4} \mathrm{SiO}_{4}, \mathrm{CO}_{3}^{2-}$ and $\mathrm{PO}_{4}^{3-}$, and other ions is very important in model simulation. The SCM parameters for metal ion adsorption on reference phases (e.g. ferrihydrite) are usually based on experimental datasets generated in simple electrolyte solutions, which is very simplistic compared to complex natural groundwater conditions. The adsorption of major ions and organic acids in natural waters is known to cause significant changes in the point-ofzero-charge and iso-electric point of mineral phases (Fuerstenau et al., 1981; Davis and Kent, 1990), so the surface reactions in the model are not truly applicable for complex electrolyte solutions such as natural groundwaters. The surface reactions used in 
the model are generated under different controlled environments in different laboratories, and under different electrolyte solutions. No evidence was found regarding the applicability of these surface reaction datasets under different redox conditions.

\section{Conclusions}

SCMs using internally consistent laboratory-derived sorption constants, calculated surface-site densities from chemical extraction data and published sorbent-site densities, measured waterrock ratios, and detailed chemical analyses of groundwater can be used to predict the distribution of As in sediments with a moderate to high level of uncertainty. The match between the modeled and extracted As by both Chao reagent and $\mathrm{HNO}_{3}$ was not successful for sediments in the deeper part of the alluvial aquifer where redox conditions are suboxic to anoxic. Within this portion of the alluvial aquifer, the reducing environment favors reductive dissolution of HFO and concomitant release of sorbed As into groundwater. In the deep alluvial aquifer, HFO is considered as a potential source for dissolved As in groundwater. The SCM was relatively successful for a shallow borehole (DRL1S), where the redox environment is relatively oxidizing to very mildly reducing, and the reductive dissolution of HFO is not an important redox process. In DRL1S, HFO in the shallow aquifer is considered as a potential sink for As.

The accuracy of the model fit depends on the qualitative and quantitative determination of accurate solid sorbent phases, selection of proper extraction methods capable of extracting the target form of solid phases, inclusion of appropriate surface parameters and internally-consistent surface reaction constants for each of the sorbent phases, and detailed and accurate chemical analysis of groundwater, including speciation of As and Fe. The level of uncertainty inherent in the predicted capacity to detect As in sediments by SCMs can be improved by accurate quantification of sorbent phases and their surface-site densities for each sediment sample included in the model. The use of default surface-site densities proposed in the literature (Dzombak and Morel, 1990; Manning and Goldberg, 1996) to calculate modeled surface-site concentrations of individual sediment samples is not applicable for the entire depth profile, because redox environments and the relative aging status of HFO phases, which control the sorption capacity, are different at different depths. Determining individual surface properties of natural sorbents at different depths within different redox environments is necessary. Developing consensus on the appropriate extraction methods and sorption databases that can be used in SCMs for natural sediments is very important.

\section{References}

Ali, M.A., Dzombak, D.A., 1996. Interaction of copper, organic acid, and sulfate in goethite suspensions. Geochim. Cosmochim. Acta 60, 5045-5053.

Allison, J.D., Brown, D.S., Novo-Gradac, K.J. (Eds.), 1991. MINTEQA2, A Geochemica Assessment Data Base and Test Cases for Environmental Systems: Ver. 3.0 User's Manual. EPA/600/3-91/-21.

Andersson, A., Nilsson, A., Hakansson, L., 1991. Metal concentrations in the mor layer. Swedish Environmental Protection Agency. Report 3990, Solna, Sweden, p. 85.

Appelo, C.A.J., Van Der Weiden, M.J.J., Tournassat, C., Charlet, L., 2002. Surface complexation of ferrous iron and carbonate on ferrihydrite and the mobilization of arsenic. Environ. Sci. Technol. 36, 3096-3103.

Arnold, T., Zorn, T., Zanker, H., Bernhard, G., Nitsche, H., 2001. Sorption behavior of U (VI) on phyllite: experiments and modeling. J. Contam. Hydrol. 47, 219-231.

Barnett, M.O., Jardine, P.M., Brooks, S.C., 2002. U (VI) adsorption to heterogeneous subsurface media. Application of a surface complexation model. Environ. Sci. Technol. 36, 937-942.

Belzile, N., Tessier, A., 1990. Interactions between arsenic and iron oxyhydroxides in lacustrine sediments. Geochim. Cosmochim. Acta 54, 103-109.

Bethke, C.M., 2006. The Geochemists Workbench, Version 6.0, A User's Guide to Rxn, Act2, Tact, React, and Gtplot. Hydrogeology Program, University of Illinois.
Brendler, V., Vahle, A., Arnold, T., Bernhard, G., Fanghanel, T., 2002. RES3TRossendorf expert system for surface and sorption thermodynamics. J. Contam. Hydrol. 61, 281-291.

Chao, T.T., Zhou, L., 1983. Extraction techniques for selective dissolution of amorphous iron oxides from soils and sediments. Soil Sci. Soc. Am. J. 47, 225232.

Coston, J.A., Fuller, C.C., Davis, J.A., 1995. $\mathrm{Pb}^{2+}$ and $\mathrm{Zn}^{2+}$ adsorption by a natural Aland Fe-bearing surface coating on an aquifer sand. Geochim. Cosmochim. Acta 59, 3535-3548.

Cowan, C.E., Zachara, J.M., Smith, S.C., Resch, C.T., 1992. Individual sorbent contributions to cadmium sorption on ultisols of mixed mineralogy. Soil Sci. Soc. Am. J. 56, 1084-1094.

Davis, J.A., Kent, D.B., 1990. Surface complexation modeling in aqueous geochemistry, mineral-water interface geochemistry. Rev. Mineral. Ser., Mineral. Soc. Am. 23, 177-260.

Davis, J.A., Coston, J.A., Kent, D.B., Fuller, C.C., 1998. Application of the surface complexation concept to complex mineral assemblages. Environ. Sci. Technol. $32,2820-2828$.

Davis, J.A., James, R.O., Leckie, J.O., 1978. Surface ionization and complexation at the oxide/water interface: I. Computation of electrical double layer properties in simple electrolytes. J. Colloid Interface Sci. 63, 480-499.

Davis, J.A., Meece, D.E., Kohler, M., Curtis, G.P., 2004. Approaches to surface complexation modeling of uranium (VI) adsorption on aquifer sediments. Geochim. Cosmochim. Acta 68, 3621-3641.

Dixit, S., Hering, J.G., 2003. Comparison of arsenic (V) and arsenic (III) sorption onto iron oxide minerals: implications for arsenic mobility. Environ. Sci. Technol. 37, 4182-4189.

Dixit, S., Hering, J.G., 2006. Sorption of Fe (II) and As (III) on goethite in single- and dual-sorbate systems. Chem. Geol. 228, 6-15.

Dzombak, D.A., Morel, F.M.M., 1990. Surface Complexation Modeling: Hydrous Ferric Oxide. John Wiley \& Sons, Toronto, Canada.

Figueira da Silva, E., 2005. Numerical modeling of arsenic transport in groundwater. Doctoral Dissertation. Univ. Michigan, Ann Arbor.

Freiwald, D.A., 1985. Average Annual Precipitation and Runoff for Arkansas, 19511980. US Geol. Surv. Water Resour. Invest. Rep. 84-4363.

Fuerstenau, D.W., Manmohan, D., Raghavan, S., 1981. The adsorption of alkalineearth metal ions at the rutile/aqueous interface. In: Tewari, P.H. (Ed.), Adsorption from Aqueous Solutions. Plenum Press, New York, pp. 3-117.

Fukushi, K., Sato, T., 2005. Using a surface complexation model to predict the nature and stability of nanoparticles. Environ. Sci. Technol. 39, 1250-1256.

Goldberg, S., 1998. Ion adsorption at the soil particle-solution interface: modeling and mechanisms in structure and surface reactions of soil particles. In: Huang, P.M., Senesi, N., Buffle, J. (Eds.), Structure and Surface Reactions of Soil Particles. John Wiley \& Sons, New York, pp. 78-408.

Goldberg, S., Glaubig, R.A., 1985. Boron adsorption on aluminium and iron oxide minerals. Soil Sci. Soc. Am. J. 49, 1374-1379.

Hemond, H.F. 1995. Movement and distribution of arsenic in the Aberjona watershed. Environ. Health Perspect. 103 (Suppl. 1), 35-40.

Honeyman, B.D., 1996. Cation and anion adsorption at the oxide/solution interface in systems containing binary mixtures of adsorbents: an investigation of the concept of adsorptive additivity. Ph.D. Thesis. Stanford Univ., CA, USA.

Joseph, R.L., 1999. Status of water levels and selected water quality conditions in the Mississippi River Valley alluvial aquifer in eastern Arkansas. US Geol. Surv. Water Resour. Invest. Rep. 99-4035, p. 54.

Kleiss, B.A., Coupe, R.H., Gonthier, G.J., Justus, B.G., 2000. Water quality in the Mississippi Embayment, Mississippi, Louisiana, Arkansas, Missouri, Tennessee, and Kentucky, 1995-1998. US Geol. Surv. Circ. 1208, 5-6.

Koretsky, C., 2000. The significance of surface complexation reactions in hydrologic systems: a geochemist's perspective. J. Hydrol. 230, 127-171.

Korte, N.E., Fernando, Q., 1991. A review of arsenic (III) in groundwater. Crit. Rev. Environ. Control 21, 1-39.

Kresse, T.M., Fazio, J.A., 2002. Pesticides, water quality and geochemical evolution of ground water in the alluvial aquifer, Bayou Bartholomew watershed, Arkansas. Arkansas Dept. of Environmental Quality. Water Quality Report WQ02-05-1, Little Rock, AR.

Kresse, T.M., Fazio, J.A., 2003. Occurrence of arsenic in groundwaters of Arkansas and implications for source and release mechanisms. Arkansas Dept. of Environmental Quality. Water Quality Report WQ03-03-01, Little Rock, AR.

Kulik, D.A., 2002. Sorption modeling by Gibbs energy minimization: towards a uniform thermodynamic database for surface complexes of radionuclide. Radiochim. Acta 90, 815-832.

Langmuir, D., 1997. Aqueous Environmental Geochemistry. Prentice Hall, Upper Saddle River, NJ, USA.

Manning, B.A., Goldberg, S., 1996. Modeling competitive adsorption of arsenate with phosphate and molybdate on oxide minerals. Soil Sci. Soc. Am. J. 60, 121131.

Manning, B.A., Goldberg, S., 1997. Arsenic (III) and arsenic (V) adsorption on three California soils. Soil Sci. 162, 886-895.

Miller, G.P., 2000. Prediction of the Environmental Mobility of Arsenic: Evaluation of a Mechanistic Approach to Modeling Water-Rock Partitioning. EPRI, Palo Alto, CA, USA. Doc. No. 1000547.

Miller, G.P., 2001. Surface complexation modeling of arsenic in natural water and sediment systems. Doctoral Dissertation. New Mexico Institute of Mining and Technology, Socorro, NM, USA.

Miller, W.P., Miller, D.M., 1987. A micro-pipette method for soil mechanical analysis. Commun. Soil Sci. Plant Anal. 18, 1-15. 
Padmanabhan, E., Mermut, A.R., 1996. Submicroscopic structure of Fe-coatings on quartz grains in tropical environments. Clays Clay Miner. 44, 801-810.

Palmqvist, U., Ahlberg, E., Lövgren, L., Sjöberg, S., 1999. Competitive metal ion adsorption in goethite systems using in situ voltammetric methods and potentiometry. J. Colloid Interface Sci. 218, 388-396.

Parkhurst, D.L., Appelo, C.A.J., 1999. User's Guide to PHREEQC (Ver. 2) - A Computer Program for Speciation, Batch-Reaction, One-Dimensional Transport, and Inverse Geochemical Calculations. US Geol. Surv. Water Resour. Invest. Rep. 99-4259.

Penn, R.L., Zhu, C., Veblen, D.R., 2001. Iron oxide coatings on sand grains from the Atlantic Coastal Plain: high resolution transmission electron microscopy characterization. Geology 29, 843-846.

Prikryl, J.D., Jain, A., Turner, D.R., Pabalan, R.T., 2001. Uranium (VI) sorption behavior on silicate mineral mixtures. J. Contam. Hydrol. 47, 241-253.

Sadiq, M., 1997. Arsenic chemistry in soils: an overview of thermodynamic predictions and field observations. Water Air Soil Pollut. 93, 117-136.

Sahai, N., Sverjensky, D.A., 1997a. Evaluation of internally consistent parameters for the triple-layer model by the systematic analysis of oxide titration data. Geochim. Cosmochim. Acta 61, 2801-2826.

Sahai, N., Sverjensky, D.A., 1997b. Solvation and electrostatic model for specific electrolyte adsorption. Geochim. Cosmochim. Acta 61, 2827-2848.

Schmeide, K., Pompe, S., Bubner, M., Heise, K.H., Bernhard, G., Nitsche, H., 2000. Uranium (VI) sorption onto phyllite and selected minerals in the presence of humic acid. Radiochim. Acta 88, 723-728.

Schrader, T.P., 2001. Status of Water Levels and Selected Water Quality Conditions in the Mississippi River Alluvial Aquifer in Eastern Arkansas. US Geol. Surv. Water Resour. Invest. Rep. 01-4124.

Sharif, M.U., 2007. Hydrogeochemical evolution of arsenic in groundwater: sources and sinks in the Mississippi River Valley alluvial aquifer, southeastern Arkansas, USA. Doctoral Dissertation. Univ. Arkansas, Fayetteville, AR, USA.

Sharif, M.U., Davis, R.K., Steele, K.F., Kim, B., Hays, P.D., Kresse, T.M., Fazio, J.A., 2008b. Distribution and variability of redox zones controlling spatial variability of arsenic in the Mississippi River Valley alluvial aquifer, southeastern Arkansas. J. Contam. Hydrol. 99, 49-67.

Sharif, M.U., Davis, R.K., Steele, K.F., Kim, B., Kresse, T.M., Fazio, J.A., 2008a. Inverse geochemical modeling of groundwater evolution with emphasis on arsenic in the Mississippi River Valley alluvial aquifer, Arkansas, USA. J. Hydrol. 350, 4155.

Shevenell, L., Connors, K.A., Henry, C.D., 1999. Controls on pit lake water quality at sixteen open-pit mines in Nevada. Appl. Geochem. 14 (5), 669-687.

Sigg, L.M., 1979. Die Wechselwirkung von Anionen und schwachen Säuren mit aFeOOH (Goethit) in wässriger Lösung. Doctoral Dissertation. Swiss Federal Institute of Technology (Eidgenössische Technische Hochschule), Zurich.

Smith, R.W., Jenne, E.A., 1991. Recalculation, evaluation, and prediction of surface complexation constants for metal adsorption on iron and manganese oxides. Environ. Sci. Technol. 25, 525-531.
Sposito, G., 1989. The Chemistry of Soils. Oxford University Press, New York.

Sracek, O., Bhattacharya, P., Jacks, G., Gustafsson, J.P., von Brömssen, M., 2004 Behaviour of arsenic and geochemical modeling of arsenic enrichment in aqueous environments. Appl. Geochem. 19, 169-180.

Sverjensky, D.A., Sahai, N., 1996. Theoretical prediction of single-site surfaceprotonation equilibrium constants for oxides and silicates in water. Geochim. Cosmochim. Acta 60, 3773-3797.

Swedlund, P.J., Webster, J.G., 1999. Adsorption and polymerization of silicic acid on ferrihydrite and its effect on arsenic adsorption. Water Res. 33, 3413-3422.

Tessier, A., Campbell, P.G.C., Bisson, M., 1979. Sequential extraction procedure for the speciation of particulate trace metals. Anal. Chem. 51, 844-851.

Tessier, A., Fortin, D., Belzile, N., Devitre, R.R., Leppard, G.G., 1996. Metal sorption to diagenetic iron and manganese oxyhydroxides and associated organic matter: narrowing the gap between field and laboratory measurements. Geochim. Cosmochim. Acta 60, 387-404.

Turner, D.R., Pabalan, R.T., 1999. Abstraction of mechanistic sorption model results for performance assessment calculations at Yucca Mountain, Nevada, USA Waste Manage. 19, 375-388.

Turner, G.D. Zachara, J.M., McKinley, J.P. Smith, S.C., 1996. Surface-charge properties and $\mathrm{UO}_{2}^{2+}$ adsorption of a subsurface smectite. Geochim. Cosmochim. Acta 60, 3399-3414.

US Geological Survey, 1994. Arsenic contamination in the Whitewood Creek-Belle Fourche River-Cheyenne River System, Western South Dakota, Bibliography of Publications from the Toxic Substances Hydrology Program. US Geol. Surv. Open File Rep. 94-91.

Van Geen, A. Robertson, A.P. Leckie, J.O. 1994. Complexation of carbonate species at the goethite surface: implications for adsorption of metal ions in natural waters. Geochim. Cosmochim. Acta 58, 2073-2086.

Waite, T.D., Davis, J.A., Fenton, B.R., Payne, T.E., 2000. Approaches to modeling uranium (VI) adsorption on natural mineral assemblages. Radiochim. Acta 88, 687-699.

Wang, F., Chen, J., Forsling, W., 1997. Modeling sorption of trace metals on natural sediments by surface complexation model. Environ. Sci. Technol. 31, 448-453.

Weirich, D., 2000. Influence of organic ligands on the adsorption of copper cadmium, and nickel on goethite. Doctoral Dissertation (ETHZ Nr. 13917). Eidgenössische Technische Hochschule (ETH), Zurich.

Westall, J., Hohl, H., 1980. A comparison of electrostatic models for the oxide solution interface. Adv. Colloid Interface Sci. 12, 265-294.

Wilkie, J.A., Hering, J.G., 1996. Adsorption of arsenic onto hydrous ferric oxide: effects of adsorbate/adsorbent ratios and co-occurring solutes. Colloid Surf. A Physicochem. Eng. Aspects 107, 97-110.

Zachara, J.M., Westall, J.C., 1999. Chemical modeling of ion adsorption in soils. In: Sparks, D.L. (Ed.), Soil Physical Chemistry. CRC Press, Boca Raton, FL, USA. 